\title{
A clinically and echocardiographically demonstrable dynamic left ventricular outflow tract obstruction
}

\author{
Saad Ahmad and Waqas Khan*
}

Ahmad S, Khan W. A clinically and echocardiographically demonstrable dynamic left ventricular outflow tract obstruction. Curr Res Cardiol 2018;5(1):1-3.

\section{ABSTRACT}

Dynamic left ventricular outflow tract (LVOT) obstruction is a distinctive finding on echocardiography and often supports a diagnosis of hypertrophic obstructive cardiomyopathy (HOCM). It is also a clinical entity in its own right and is reproducible with stress. We describe a case of dynamic outflow obstruction in an elderly man with classical exertional symptoms. Echocardiography was used to demonstrate a variable gradient, with imaging performed at rest and following physical exertion. We will review the pathophysiological changes seen in this condition, both in terms of cardiac structure and the physics of fluid dynamics. Medical treatment is centered around a meaningful understanding of these changes, but there are also surgical and ablative options available. Device therapy with an implantable cardioverter-defibrillator (ICD) is the mainstay of HOCM treatment, particularly when ventricular arrhythmias are evident. A risk stratification for sudden cardiac death from hypertrophic cardiomyopathy is imperative in case by case management.

Key Words: Echocardiography; Hypertrophic cardiomyopathy; Left ventricular outflow tract; Dynamic; Cardiac structure

\section{CASE REPORT}

$\mathrm{A}^{78}$ 78-year-old gentleman with atrial fibrillation, hypertension and diabetes presented to our hospital with chest pain and exertional dyspnoea. He was investigated, and significant acute respiratory or cardiac pathology was excluded. He was however noted to have a high left ventricular outflow tract (LVOT) gradient on trans-thoracic echocardiography and an abnormal baseline ECG (Figures 1 and 2). He was monitored and found to have breathlessness on minimal exertion. At rest, a full cardiac examination was relatively unremarkable with no audible murmurs. A repeat examination following a short walk down the ward corridor, revealed a harsh ejection systolic murmur on auscultation. This was heard throughout the precordium but loudest in the aortic region with radiation to the carotid arteries. There was an associated ventricular heave.

A further echocardiogram was performed after a modified exercise test. This was a focused study to assess the LVOT and cardiac septum. The initial echocardiogram had shown turbulent flow in the LVOT and across the aortic valve. There was basal septal hypertrophy with a peak gradient of 31 $\mathrm{mmHg}$. The repeat scan post-exercise demonstrated a significant increase in the gradient to $104-114 \mathrm{mmHg}$. These findings were diagnostic of a dynamic LVOT obstruction, likely to be in association with Hypertrophic Obstructive Cardiomyopathy (HOCM) (Figures 3-5).

There had been no prior history of serious arrhythmias or syncope and certainly nothing untoward was found over several days of cardiac monitoring with telemetry. Therefore, considering this gentleman's age and low risk of sudden death, he was managed with B-Blocker therapy. He was referred to a tertiary center for consideration of septal ablation or myomectomy.

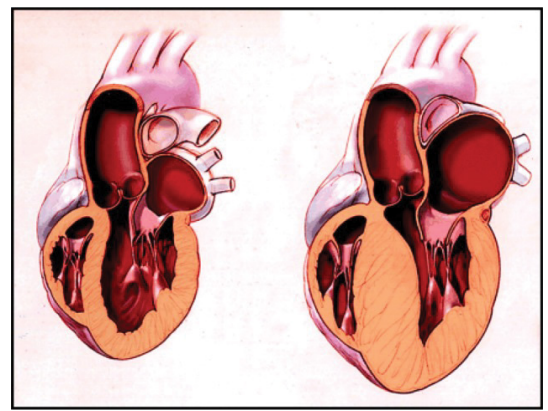

Figure 1) An illustration of a heart with hypertrophic cardiomyopathy on the right in comparison to a normal heart on the left

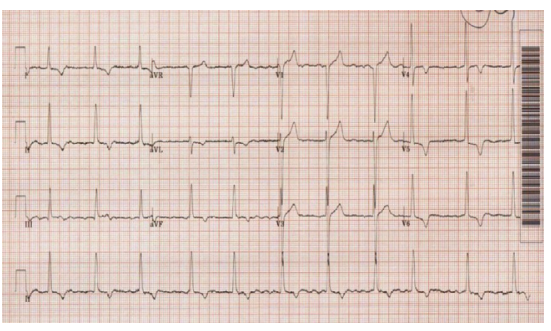

Figure 2) Our patient's resting 12-lead ECG showing atrial fibrillation, high amplitude QRS complexes and deep T wave inversion inferolaterally

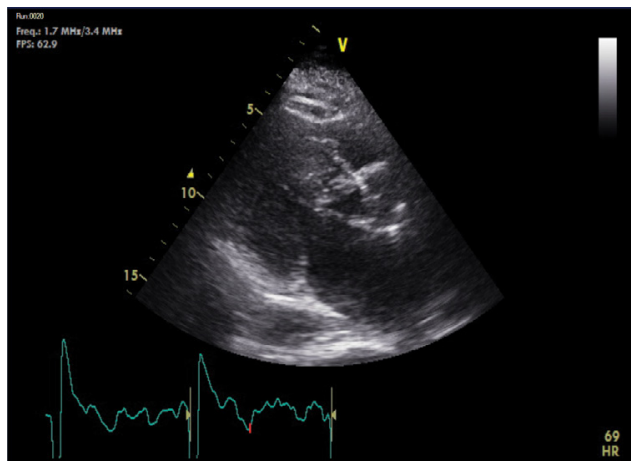

Figure 3) Dynamic LVOT obstruction at end-systole

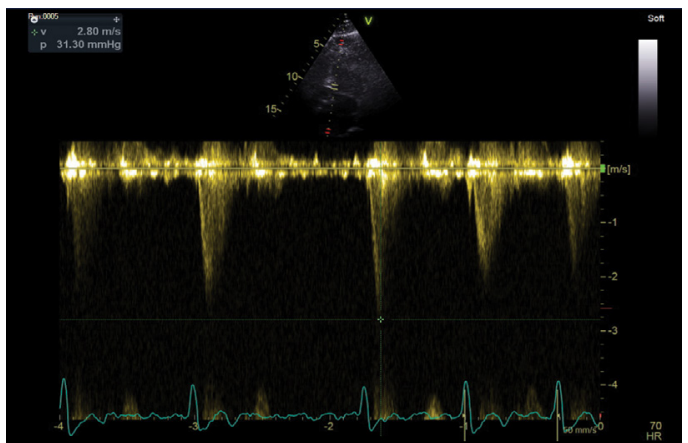

Figure 4) Resting LVOT gradient $(31.30 \mathrm{mmHg}, 2.8 \mathrm{~m} / \mathrm{s}$ )

Department of Cardiology, Royal Preston Hospital, United Kingdom

Correspondence: Dr Waqas Khan, Department of Cardiology, Royal Preston Hospital, United Kingdom, Telephone 441772 716565, e-mail Waqas.Khan@lthtr.nhs.uk Received: November 27, 2017, Accepted: January 03, 2018, Published: January 05, 2018 


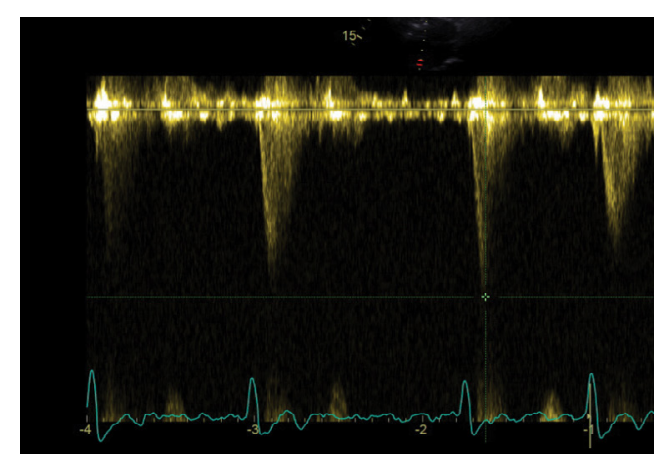

Figure 5) Post-exercise LVOT gradient $(114.40 \mathrm{mmHg}, 5.35 \mathrm{~m} / \mathrm{s})$

\section{DISCUSSION}

Hypertrophic Cardiomyopathy (HCM) is an inherited cardiac muscle disorder resulting in a structurally and often functionally abnormal heart. An illustration of the structural changes is shown in Figure 1. Though inheritance is primarily autosomal dominant, it is a heterogeneous condition for which over 150 gene mutations have been described. For this reason, there is variability in the presentation of any given case of HCM, i.e., the clinical phenotype reflects variable penetrance (1).

The cardiac septum and apex are often grossly thickened leading to left ventricular hypertrophy (LVH). The hypertrophied muscle can be arrhythmogenic and a focus for ventricular arrhythmias in sudden cardiac death. Indeed, HCM is a prominent cause of sudden death in athletes with an annual mortality of approximately $1-2 \%$ (2). Asymmetrical thickening of the anterior portion of the septum is the usual observed pattern, associated with systolic anterior motion (SAM) of the mitral valve and left ventricular outflow tract (LVOT) obstruction (3). If a dynamic obstruction is evident, hypertrophic cardiomyopathy is often referred to as hypertrophic obstructive cardiomyopathy (HOCM).

The clinical features in HCM include chest pain and shortness of breath, such as those seen in our patient. These symptoms are often progressive and exertional, in line with the physiological changes that occur during activity. Dynamic, exertion-related LVOT obstruction appears to be the underlying reason for these symptoms and is precipitated under stress when heart rate and cardiac output need to increase to match bodily demands (4). Presyncope and syncope are other symptoms explained by outflow obstruction. Palpitations can also occur due to arrhythmias and heart failure symptoms of orthopnoea and fatigue can be seen if there is left ventricular dysfunction. Diastolic dysfunction is more prevalent in HCM as the muscle is stiff and fails to relax normally with impaired filling (5).

Electrocardiography (ECG) can be useful as a screening test for suspected cardiomyopathy. Increased QRS voltage in precordial leads is indicative of LVH. Q waves in inferolateral leads and non-specific ST segment and T wave abnormalities are also suggestive. In particular, widespread deep T wave inversion is a commonly encountered finding, especially in apical disease and as seen in our case. Supraventricular arrhythmias and atrial fibrillation can also be seen. There is an association between Wolff-Parkinson-White (WPW) syndrome and HCM and this has been described in literature.5 Ventricular arrhythmias are prognostically significant and the search for these requires ambulatory ECG monitoring. All patients suspected to have cardiomyopathy should at the very least undergo a 24-hour tape to uncover asymptomatic non-sustained ventricular tachycardia (NSVT), the presence or absence of which has a bearing on treatment. Detailed assessment of cardiac structure entails investigation with Echocardiography and Cardiac MRI. Echocardiography can quantify the extent and distribution of hypertrophy. The maximum left ventricular (LV) wall thickness and outflow tract gradients are two vital parameters. When non-invasive transthoracic images are suboptimal, TOE and Cardiac MR (CMR) may be pursued in selected patients in order to accurately obtain these measurements. Furthermore, the left atrium (LA) is often enlarged in cardiomyopathy and the size of this chamber is prognostically significant. The mechanism predisposing to dilatation is usually a combination of elevated LV filling pressures and SAM-related mitral regurgitation, a concept which will be discussed in the next section.

Exercise stress echocardiography was performed in our case and is a recommendation as set out by the European Society of Cardiology (ESC). Symptomatic patients should have exercise echocardiography if bedside manoeuvres fail to induce a LVOT gradient $>50 \mathrm{mmHg}$. As for asymptomatic individuals, routine exercise stress echocardiography has not been prospectively evaluated but may be useful in risk stratification. CMR is another commonly performed investigation in hypertrophic cardiomyopathy. Although it provides similar information as transthoracic echocardiography in terms of ventricular morphology and function, it is superior in delineating anterolateral and apical disease. Furthermore, CMR may be helpful in surgical work-up for myomectomy, particularly where there is a multi-level obstruction at the mid-cavity and at the outflow tract. It also has a role following septal alcohol ablation as it can assess the extent of tissue necrosis and regression of LV mass after the procedure.

Asymmetry of the interventricular septum is the pathophysiological basis for a dynamic obstruction in the left ventricular outflow tract. Accelerated blood flow is triggered by hyperdynamic circulation and hypertrophy of the septum (6). The anterior mitral valve leaflet and septum constitute the outflow tract and it is the relationship between the two that is integral to understanding the mechanism of a dynamic obstruction.

Applying a concept of fluid dynamics, the velocity of an incompressible fluid like blood must increase as it makes its way through a constriction. Conversely, the pressure of that fluid must reduce in accordance to conservation of mechanical energy. This is the principle behind the Venturi effect (6). Therefore, the narrowed LVOT passage in HOCM makes for greater velocity but lower pressure. The low pressure pulls the anterior mitral valve leaflet in the direction of the interventricular septum, a phenomenon described as Systolic Anterior Motion (SAM) (3,7). In turn, this constricts the LVOT diameter further. Studies and observation have demonstrated that SAM occurs when there is a dynamic LVOT obstruction8, such as in the case of our patient. The traction on the mitral valve can inevitably cause a degree of functional disruption and mitral regurgitation.

Bearing in mind physiological variables and the physics discussed above, it is important to remember there are factors that can impact the LVOT gradient. Ventricular contractility can worsen the degree of obstruction (8). A reduction in preload and afterload and a smaller ventricular cavity are other potential exacerbating factors (7). Treatment is aimed at ameliorating such variables. A volume status assessment and correction of a hypovolemic state is imperative. Inotropes are best avoided so as to keep ventricular contractility in check. Such is the rationale behind the use of rate limiting drugs like beta-blockers and calcium channel antagonists as negatively inotropic agents, particularly in HOCM. Nonetheless, a risk stratification should take place in all cases (9). An implantable cardioverter defibrillator is indicated if there is evidence for ventricular arrhythmia or a high risk of sudden cardiac death $(5,10)$. Surgical and ablative strategies are considered when providing symptomatic relief, especially in cases with significant LVOT obstruction. Conventionally, the threshold for invasive treatment is usually considered to be $>50 \mathrm{mmHg}(11)$.

Risk assessment for Sudden Cardiac Death (SCD) may be adopted from the European Society of Cardiology (ESC) guidelines (11). This takes into account the patient's history and clinical features as well as findings on echocardiography. The three main clinical factors of concern are a (a) family history of sudden cardiac death, (b) unexplained syncope and (c) evidence of ventricular arrhythmia. In conjunction with the patient's age and echocardiographic parameters including maximum LVOT gradient, maximum LV wall thickness and LA size, a 5-year risk of death can be deduced. In fact, there is a web-tool available for risk calculation that also provides ESC recommendation with regards to ICD implantation. In our patient's case, risk percentage was only $1.69 \%$ and as such an ICD was not generally indicated.

\section{CONCLUSION}

In usual clinical practice, not often is a cardiovascular examination performed after exertion and at rest. An important clinical sign can therefore be missed in cases of dynamic LVOT obstruction. There may be an argument to encourage more vigorous assessment and this is in accordance with ESC guidance whereby examination on exertion is recommended as part of a full physical examination. The Valsalva manoeuvre is a technique that reduces ventricular preload and afterload and can precipitate a murmur or indeed make it more audible. A short walk down the hospital corridor can serve a similar purpose, especially in cases of HCM or dynamic LVOT obstruction.

Management of hypertrophic cardiomyopathy is individualized and dependent on risk as described above. Regular echocardiographic surveillance is key and other imaging modalities such as cardiac MR have a role to play. A holistic approach should always be adopted, not taking for granted family genetics and the counselling aspects surrounding a diagnosis of HCM. Medical treatment with B-Blockers is almost universal. Close follow-up and continuous risk stratification are essential prior to ICD implantation and other invasive measures. 


\section{REFERENCES}

1. Maron BJ, Maron MS, Semsarian C. Genetics of hypertrophic cardiomyopathy after 20 years: Clinical perspectives. Journal of the American College of Cardiology 2012;60(8):705-15.

2. Shoji M, Uejima T, Matsuno S, et al. Dynamic narrowing of the left ventricular outflow tract - Possible mechanism of latent left ventricular outflow tract obstruction. Journal of Cardiology Cases 2010;2(2):74-7.

3. Mintz GS, Kotier MN, Segal BL, et al. Systolic anterior motion of the mitral valve in the absence of assymetrical septal hypertrophy. Circulation 1978;57:236-56.

4. Sharma R, Pellerin D, Gaze DC, et al. Dynamic left ventricular obstruction: a potential cause of angina in end stage renal disease, Int J Cardiol 2006;112:295-301.

5. Houston BA, Stevens GR. Hypertrophic cardiomyopathy: A review. Clin Med Insights Cardiol 2014;8:53-65.
6. Turnage WS, Zeller FA, Divertie GD. Dynamic left ventricular outflow tract obstruction. Diagnosis by transoesophageal echocardiography in a critically ill patient. November 1993;104(5):1629-31.

7. Bulkley BH, Fortuin, NJ. Systolic anterior motion of the mitral valve without asymetric septal hypertrophy. Chest 1976;69:694-6.

8. Come PC, Bulkley BH, Goodman ZD, et al. Hypercontractile cardiac states simulating hypertrophic cardiomyopathy. Circulation 1977;55:901-8.

9. Hansen MW, Merchant N. MRI of hypertrophic cardiomyopathy: part2, Differential diagnosis, risk stratification, and post treatment MRI appearances.

10. Mookadam F, Haley JH, Olson LJ, et al. Dynamic left ventricular outflow tract obstruction in senile cardiac amyloidosis. European Journal of Echocardiography 2006;7(6):465-8.

11. Elliot PM, Anastasakis A, Borger MA, et al. 2014 ESC Guidelines on diagnosis and management of hypertrophic cardiomyopathy. European Heart Journal 2014;35:2733-79. 